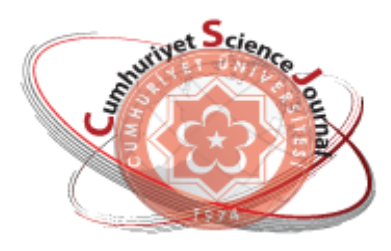

e-ISSN: $2587-246 X$

ISSN: $2587-2680$

\section{Cumanoryot Seience Journal $\cos$}

Cumhuriyet Sci. J., Vol.38-3 (2017) 444-449

\title{
Electric Quadrupole Transitions in Processes With Stripping Bineutrons Clusters
}

\author{
I.G. AFANDIYEVA \\ Azerbaijan State University of Oil and Industry Baku, AZERBAIJJAN \\ Received: 17.04.2017; Accepted: 17.07.2017 \\ http://dx.doi.org/10.17776/csj.340478
}

\begin{abstract}
Based on the theory of clusters discussed the electric quadrupole transitions in two-nucleon transfers as $(t, p)$. The study did not take into account the interaction between the particles of the association. The internal structure of the clusters is neglect and effects of the Pauli principle between the nucleons in the associations are taken into account by introducing short range repulsion between the associations. Study was carried out with the distorted plane waves of proton and of triton. The results are given in the work related to the analysis of the matrix element of the electric quadruple transitions. This report demonstrated via some examples how the nuclear magnetic and quadrupole moment are sensitive to some features in the nuclear structure.
\end{abstract}

Keywords: Two-nucleon transfers

\section{Sıyırma Bineutronları Kümeleri ile İşlemlerdeki Dört kutuplu Elektrik Geçişleri}

Özet: Kümeler teorisine dayanarak, iki-çekirdekli transferlerdeki dört kutuplu elektrik geçişleri (t, p) olarak tartışılmıştır. Çalışma, parçacıklar arasındaki etkileşimi hesaba katmamıştır. Kümelerin iç yapısı ihmal edlmiştir ve dernekler arasında kısa menzilli iticilik getirerek nükleonlar arasındaki Pauli ilkesinin etkileri dikkate alınmıştır. Çalışma, proton ve tritonun çarpık düzlem dalgaları ile uygulanmıştır. Sonuçlar, dört kutuplu elektrik geçişlerinin matris elemanlarının analizi ile ilgili çalışmada verilmişstir. Bu rapor, nükleer manyetik ve dört kutuplu momentin nükleer yapıdaki bazı özelliklere nasıl duyarlı olduğunu bazı örnekler vasıtasıyla sunmaktadir.

Anahtar Kelimeler: İki çekirdekli transferler

\section{INTRODUCTION}

The idea of nucleons clustering has a history back to the early thirties of the last century. By observing alpha decay from nucleus, people speculated that nuclei are made up of alpha particles. Bethe in his paper [1] predicted that nuclei are made of alpha particles and gave also a geometrical arrangement of alpha particles inside nuclei. He predicted that, for each new addition of alpha particles, the number of bonds increased is three. At that time, this was too a big challenge for the clustering models and eventually these models disappeared from the nuclear structure theories. Clustering is a recurrent feature in light nuclei, from beryllium to nickel. In light nuclei, the nucleons have been observed to cluster together forming substructures within the atomic nucleus, for states where the nucleons are only just bound together. Cluster structures are typically observed as excited states close to the corresponding decay threshold; the origin of this phenomenon lies in the effective 
nuclear interaction, but the detailed mechanism of clustering in nuclei has not yet been fully understood. If we are taking into account the interaction between nucleons (as it is in reality) in the model approach, we can expect the formation of clusters. Calculations show that, the associations occur on the surface of the nucleus, where the density of nuclear matter less than in the downtown nucleus [2].

Since the formation of associations, most likely on the surface area of the nuclei, then to study them in the first place it is necessary to carry out studies of nuclear processes, extending to the surface of the nucleus. These are direct nuclear reactions. The association effect is significant when the scattering particles with light nuclei have intermediateenergy and it appears the stronger the higher the energy of the incident particle. The information obtainable from bineutron cluster stripping reactions is different from that obtained from single-nucleon transfer reactions; from the latter only the single particle character of the levels is obtained. The sets of data obtained from the single and two nucleon transfer reactions are both valuable, and they are mutually complementary. The analysis of the former kinds of reactions has already reached a satisfactory enough stage to furnish quantitative information about nuclear structure.

The two-nucleon transfer reactions also, have taken on great importance, because they are to be very sensitive to nuclear spectroscopy. The task of the proton-triton or triton-proton scattering is the simplest problem in the nucleon - nucleus interaction or interaction of a particle with a complex structure, which has been studied for a long time and its solution, developed highly effective calculation schemes.

The information obtainable from bineutron cluster stripping reactions is different from that obtained from single-nucleon transfer reactions; from the latter only the single particle character of the levels is obtained. The sets of data obtained from the single and two nucleon transfer reactions are both valuable, and they are mutually complementary.
The analysis of the former kinds of reactions has already reached a satisfactory enough stage to furnish quantitative information about nuclear structure.

In this paper, we obtained the expression for matrix element for electric quadruple transition. Considered energy region lies below the threshold meson production and the impulse approximation is used. It is assumed that the amplitude of the interaction of nucleons of the incident nucleus with the nucleons of the target nucleus are the same as in the case of a collision free nucleons.

\section{MATRIX ELEMENT OF ELECTRIC QUADRUPLE TRANSITIONS}

Consider the $t+A \rightarrow B+p$ reaction in the laboratory system and we will study the knocking out bineutrons cluster. Let $\vec{r}_{p}, \vec{r}_{n_{1}}$ and $\vec{r}_{n_{2}}$ coordinates of the proton and neutron in the triton, $\vec{k}_{p}$ - free proton wave vector, $\vec{k}_{t}$ - wave vector of triton. In $(t, p)$ reactions occur dissipative phenomena, i.e. the dissipation in the orbital angular momentum of a reaction in which part of the relative angular momentum of $\mathbf{L}_{\mathbf{i}}$, characterizing the initial trajectory, transform into spin of fragments in accordance with the expression $\Delta \mathbf{L}=\mathbf{L}_{\mathbf{A}}-\mathbf{L}_{\mathbf{t}} \equiv \mathbf{L}_{\mathbf{B}}+\mathbf{L}_{\mathbf{p}}$.

We will neglect the internal structure of the associations and effects of the Pauli principle between the nucleons in the clusters are taken into account by introducing short range repulsion between the clusters. The orthogonality condition model and excluded state model treat the clusters as elementary particles, but include effects of the Pauli principle in a more microscopic way. Wheeler's resonating group method is a fully microscopic theory for calculating properties of cluster systems. It makes simplifying assumptions about the internal structure of the clusters but takes the Pauli principle explicitly [3].

Scattering may be accompanied by transitions between different states of the $L J$, compatible the conservation the total angular momentum. However, the orbital angular momentum and spin 
are not good quantum numbers and the partial wave corresponding to a certain $L$ and $S$, may cause scattered wave with $L^{\prime} \neq L, S^{\prime} \neq S$. If we take into account that the good quantum numbers are the total angular momentum and parity, the amplitude of the scattered waves form a matrix, the elements of which depend on the following indices: $J, L$ and $S$.

In the scheme of connection $\mathbf{I}_{\mathbf{t}}+\mathbf{L}=\mathbf{j}, \mathbf{I}_{\mathbf{A}}+\mathbf{j}=\mathbf{J}$, due to the statistical nature of the dissipation of angular momentum transmitted $\Delta \mathbf{L}$ not completely polarized, and the back can be decomposed into alignment $\boldsymbol{J}$ and fluctuation $\boldsymbol{J}^{\prime}$ components, ie, from $\left\langle J_{x}\right\rangle=\left\langle J_{y}\right\rangle=0,\left\langle J_{z}\right\rangle=J_{0}$.

The transmitted $\Delta \mathbf{L}$ orbital angular momentum is basically alignments and increases with the loss of kinetic energy. This transfer of angular momentum takes place in two ways: as the inelastic excitation of core as certain multipole and nucleons occupying certain orbital in the initial and the residual nucleus. Thus it is possible to put the physical problems of the dissipation of energy and angular momentum on the distribution $\Delta \mathbf{L}$ between fragments and the orientation of each fragment, characterized by the ratio $\mathrm{J} / J^{\prime}$ depending on such typical reaction parameters such as energy, deflection angle.

This is an illustration of the fact that the clusters of the nucleons are compatible with the Young scheme of the orbital part of the wave function if this scheme contains the maximal number of fourgroups.

The wave function of the initial state can be written as [4]

$\Psi_{i}=\Psi_{A}(\xi) F_{t}\left(\vec{r}_{t}\right) f^{S}(\vec{r}, \vec{\rho}) \chi_{1 / 2, m_{t}}$,

where $\Psi_{A}(\xi)$ the wave function of the nucleus $A$, $F_{t}\left(\vec{r}_{t}\right)$ - distorted wave function of the triton, $\vec{r}_{t}$ the radius vector in the centre of inertia of triton, $f^{s}(\vec{r}, \vec{\rho})$ - the wave function of the internal motion of triton, $r=\left|\vec{r}_{n_{1}}-\vec{r}_{n_{2}}\right|, \quad \vec{\rho}$ - distance from the proton to the centre of gravity bineutrons cluster, $S$ indicates the spin state of the captured neutrons, $\chi_{1 / 2, m_{t}}$ - spin function of the triton. Bineutron association in the triton is different from free nucleons, as they are surrounded by other nucleons. This field leads to a change in the association properties compared with the free neutrons [5].

The wave function of a nucleus $A$ consisting of $N$ bineutron clusters has the form:

$$
\Psi_{A}=\hat{A}\left\{P_{i}\left(R_{1} \ldots R_{N}\right) \exp \left[-\frac{a}{2} \sum_{i=1}^{N} \sum_{j=1}^{2}\left(r_{i j}-R_{i}\right)^{2}-2 b \sum_{l=1}^{N} R_{l}^{2}\right] \chi(\boldsymbol{\sigma \tau})\right\}
$$

where $\hat{A}=\sum_{p}(-1)^{p} P$ - the antisymmetrization operator (here the sum over all $A$ ! permutations of the nucleons of the nucleus); $P_{i}$ is a polynomial of degree $n_{i}$ that defines the shell configuration of the nucleus, parity, total orbital angular momentum, its projection, etc. of the state; $\chi(\boldsymbol{\sigma \tau})$ - spin-isospin function; $\mathbf{r}_{\mathrm{ji}}$ is the radius vector of the $j$-th nucleon in the $l$-th binucleon association; $R_{l}=\frac{1}{2} \sum_{j=1}^{2} r_{j l}$ and $x=b / a$ parameter of isolation. For $\mathrm{x} \rightarrow 1$, the wave function goes over into the shell function.

The final state function describes the state of the nucleus $B$ consisting of $A$ and bineutrons cluster and the free movement of a proton. It can be represented as:

$\Psi_{f}=\Psi_{B}(\xi) f\left(\vec{r}_{p}\right) \chi_{1 / 2, m_{p}}$,

where $f\left(\vec{r}_{p}\right)$ - distorted function of the proton, $\chi_{1 / 2, m_{p}}$ - the spin function of the proton.

The matrix element of the quadrupole transition has the following form

$M=\frac{\left\langle\Psi_{i}|\hat{Q}| \Psi_{j}\right\rangle}{\sqrt{N_{i} N_{j}}}$,

where 
$\hat{Q}=\sum_{i=1}^{A} r_{j}^{2} Y_{20}\left(\theta \varphi_{i}\right)$

- quadrupole moment operator; $N_{i}$ and $N_{j}$ the normalization integrals.

The main feature of the integrals appearing in expressions (4) is that the wave function of the relative movement and distorted waves also depend on the relative different combinations of variables. Furthermore, they are presented in nucleon variables. The problem of separation of variables is very important because of its solution depends on the possibility of analytical calculation of integrals over the angular variables, as well as for those variables that are not associated with the interaction of particles.

The operator $\hat{Q}$ is not symmetric in all nucleons, since it acts only on the variables of the protons. Taking into account the contribution of the spin or isospin part of the operator $\mathrm{G}$ does not cause any special difficulties. This problem is solved in the same way as in the shell model.

The quadrupole transition operator in the Jacobi variables can be written as follows:

$$
\begin{aligned}
& \hat{Q}=2 R^{2} Y_{20}(r) \\
& +\sum_{l=1}^{A}\left\{r_{l}^{2} Y_{20}\left(r_{l}\right)+\frac{1}{2} u_{l}^{2} Y_{20}\left(u_{l}\right)+\frac{1}{2} v_{l}^{2} Y_{20}\left(v_{l}\right)\right\}
\end{aligned}
$$

where variables the Jacobi variables describe the motion of bineutrons inside the $l$ cluster:

$u_{l}=r_{l 1}-r_{l 2} ; v_{l}=r_{l 1}+r_{l 2}$.

The term containing the quadrupole moment of the center of mass of the nucleus is discarded, since it does not correspond to any real physical transition of the nucleus from one state to another, but leads to excitation of the center of mass of the nucleus.

Since antisymmetric functions are in the left and right side of the integral and the operator $\hat{Q}$ is symmetric with respect to the nucleons, then we can write
$M=\frac{A !\left\langle\hat{A} \Psi_{i}|\hat{Q}| \Psi_{j}\right\rangle}{\sqrt{N_{i} N_{j}}}$.

Taking into account the form of the operator $\hat{Q}$, it is possible to represent (8) in the form $A$ ! of terms, one of which is a direct integral and has the form $\left\langle\Psi_{i}|\hat{Q}| \Psi_{j}\right\rangle$, and the remaining exchange ones -

\section{$\left\langle P \Psi_{i}|\hat{Q}| \Psi_{j}\right\rangle$.}

Let us consider the calculation of the matrix element for the quadruple transition $0^{+} \rightarrow 2^{+}$in ${ }^{234} \mathrm{U}(\mathrm{t}, \mathrm{p}){ }^{236} \mathrm{U}$ :

$M(E 2)=\frac{74 !\left\langle\hat{A} \Psi_{00}|\hat{Q}| \Psi_{20}\right\rangle}{\sqrt{N_{00} N_{20}}}$,

where

$$
\begin{aligned}
& \Psi_{00}=\left\{\exp \left[-\frac{a}{2} \sum_{i=1}^{N} 74 \sum_{j=1}^{2} \frac{1}{2}\left(u_{i j}^{2}+v_{i j}^{2}\right)-\frac{1}{2} b \sum_{l=1}^{N} R_{l}^{2}\right] \chi(\vec{\sigma}, \vec{\tau})\right\} x, \\
& x F_{t}\left(\vec{r}_{t}\right) f^{S}(\vec{r}, \vec{\rho}) \chi_{1 / 2, m_{t}}
\end{aligned}
$$

and

$$
\Psi_{20}=\Psi_{20}(\xi) f\left(\vec{r}_{p}\right) \chi_{1 / 2, m_{p}} .
$$

In view of the fact that a large number of singleparticle states are taken into account in calculations, the effective charge for bineutrons is assumed to be zero for quadrupole transitions.

Substituting (10) and (11) into (9) for the matrix element, we obtain for direct integral

$$
\begin{aligned}
& M(E 2)=\pi^{2} 2^{-\left(L+2+\frac{n+n^{\prime}}{2}\right)}\left(1-\frac{q^{2}}{4 s p}\right)^{-1 / 2\left(3+n+n^{\prime}\right)} \\
& F\left[\frac{1}{2}(L-n) ; \frac{1}{2}\left(L-n^{\prime}\right) ; L+3 / 2 ; \frac{q^{2}}{4 s p}\right] / x^{71 / 2}
\end{aligned}
$$

In the denominator of the (12) appears parameter of isolation $x^{71 / 2}, 71 / 2=\left(3 N+n_{1}+n_{2}+n\right), N=22$ the number of associations in the nucleus, $n_{1}$ and the $n_{2}$-degree of the polynomials in the functions and $n$ the rank of the operator. 
Thus the strong population in two nucleons reactions of the excited states in the actinide region can be associated with the density distribution of single - particle levels and the alignment of the corresponding quadrupole moments in the vicinity of the Fermi surface of these nuclei.

In Fig. 1 shows the $M(E 2)$ value for transition from the two excited to the ground state. The solid line corresponds to the matrix element calculated by Eq. (12) point data from [6]. Breakdown of the E2additivity for strongly interacting protons and neutrons. Only in the weak coupling limit it is possible to describe the empirical quadrupole moment for a composed proton-neutron state as a linear combination of the respective proton- and neutron-quadrupole moments. If the experimental quadrupole moment of a composed state does not agree with the empirical additivity value, it can be an indication that there is a strong interaction between the protons and the neutrons. In that case, the wave function cannot be decoupled into its proton and neutron part only. In this paper all the calculations were based on non-relativistic quantum mechanics. But at sufficiently high energies become significant inelastic processes associated with the formation of mesons.

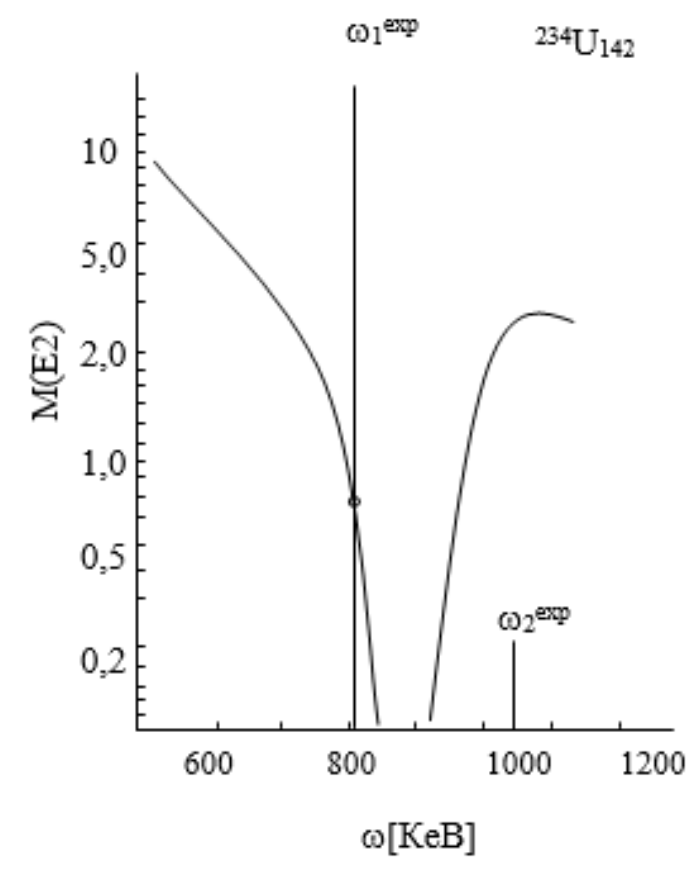

Figure 1. Shows the $M(E 2)$ value for transition from the excited to the ground state. The solid line corresponds to the matrix element calculated by Eq. (12) point data from [6].
The problem, in the solution of such problems is the separation of variables, since it affects the possibility of analytic computation of integrals over the angles, as well as for those variables that are not related to the interaction of particles. Therefore, the ability to describe the interaction between nucleons via potential is limited by high energy. Such a restriction on energy means that the unambiguous definition of the potential at small distances impossible.

\section{CONCLUSION}

For an explanation of two-nucleon transmission there are different approaches, each of which is treated properly, some but not all aspects of these transitions. Among these aspects: the use of the exact wave functions of triton and the corresponding exact interaction; accurate interpretation of the limited domain of interactions in the amplitude of both the first and second order; explanation of non-orthogonality of the amendment to the term of the second order, which seeks to eliminate the amplitude of the first order. In addition, all the results are very sensitive to potentials and the wave functions of the kernel used for the transition matrix.

The article was used approaching zero radiuses with the assumption that there is not take into account the exchange of nucleons between the two nuclei, and that the target nucleus is not excited. Then, we were limited by the fact that the internal states incident $t$, emitted $p$, and any intermediate state associations are fully balanced $S$-states, so that the corresponding sequential interaction of transmission are diagonal in the spin states of nuclei. With this reasoning radial wave functions two neutrons which form association close to one another, particularly in the peripheral region of the nucleus where there is a "cross-linking" of the wave functions of nucleons - target $(A)$ and in the nucleus $B$. 
However, we would like to emphasize once again that before we modify the nuclear model it is necessary to investigate the reliability of the spectroscopic factors extracted from the relative excitation cross sections in the reactions of twonucleon transfers, that is, to investigate the mechanism of reaction. In the two-nucleon transfer reactions, the low-lying $0^{+}$levels will be more strongly excited in nuclei in which the singleparticle quadrupole transitions near the Fermi surface have the same sign.

This report demonstrated via some examples how the nuclear quadrupole transitions are sensitive to some features in the nuclear structure.

\section{REFERENCES}

[1]. Freer M.// Scholarpedia 2010, V 5, p. 965.

[2]. Jenkins D., Courtin S// J. Phys. G: Nucl. Part. Phys. 2010, 42, 034010.

[3]. Brink D. M.// J. Phys.: Conf. Ser. 2008, 111, 012001.

[4]. Abdulvahabova S.G., Ahmedov R.A., Afandiyeva I.K. Journal of Physical Sience and Aplication. (US), 2015, V 5, N.2, p 158-162.

[5]. Y. A., Berejnoy, V. P Mikhayluk. "Alpha Clustering in the Processes Scattering Particles and Light Nuclei by Nuclei." 2008. PEPAN 39 (2): 437.

[6]. J.V. Maher et al. Phys. Rev. Lett. 1990, 25, p. 302 